\title{
Ergonomics Risk-Assessment Methods in Shipbuilding: Application of OWAS Considering Work load and Frequency for Risk Factor Analysis of WMSDs
}

\author{
Jun-Youb Lee ${ }^{1}$, Hyun-Soo Seo ${ }^{2}$ \\ ${ }^{1}$ Industrial-Hygiene Manager, Gunsan HSE Department, Hyundai Heavy Industries Co.Ltd, \\ ${ }^{2}$ Industrial-Hygiene Manager, HSE Department, Hyundai Heavy Industries Co.Ltd
}

\begin{abstract}
Objective: The aim of this paper is to introduces Hyundai Heavy Industry's ergonomic risk assessment tool, H-OWAS, which is considering work load and frequency compared to the OWAS. Background: As prevalence of work-related musculoskeletal disorders(WMSDs) in ship building industries has been much higher than that in other industries, most of the large scaled corporations have implemented their own prevention program since legislation on the prevention of WMSDs had introduced in 2003. Method: This paper introduces WMSDs prevention program, HEMP(HHI Ergonomics Management Program) which consists of risk assessment, improvement efforts of working environments, medical treatment and training/evaluation and describes how to operate the program. We also describe application of OWAS method considering work load and frequency for risk factor analysis(H-OWAS) and shows methodology for assessing the ergonomic risk factor. And comparison of the assessment results between OWAS and H-OWAS is carried out by statistical analysis. Result: There was statistically significant difference in the assessment results between OWAS and H-OWAS, and regression shows H-OWAS explains the borg's scale of perceived exertion more clearly than OWAS. Conclusion: H-OWAS has been proved more effective tool than OWAS to evaluate ergonomic risk factor under real working condition. Application: H-OWAS can be widely applied to the many other companies when implementing the ergonomics risk assessment.
\end{abstract}

Keywords: Ergonomic, H-OWAS, Risk factor analysis, WMSDs

\section{Introduction}

사업장 근골격계질환 증가에 따른 정부 차원의 대책의 일 환으로 2003년부터 법제화를 통해 유해요인조사, 작업환경 개선, 의학적 관리 및 이를 포함한 예방관리 프로그램의 시 행 등 근골격계 부담작업 보유 사업장에 대한 예방관리가 의무화된 이후, 많은 기업에서 자사의 근골격계질환 예방관 리 체계를 확립하는데 많은 노력을 기울여 왔다. 특히 조선업 은 대표적인 근골격계질환 다발 업종인 만큼 근골격계질환
예방관리 프로그램 법적 시행대상이 되는 대규모 사업장의 경우 외부 전문가 영입 및 자체인력 양성 등 전문인력 확보 를 통한 체계적이고 효율적인 시스템 구축으로 많은 성과를 보이고 있다. 본 논문은 세계 제 1 위 조선업체인 $\mathrm{H}$ 社의 근 골격계질환 예방관리 프로그램인 $\mathrm{HEMP}(\mathrm{HHI}$ Erogonomics Management Program) 시스템을 소개하고 이의 일환으로 시행중인 인간공학적 Risk Assessment 방법론에 대해 소 개하고자 한다. 통상적으로 법에서 정한 법정 유해요인조사 의 경우 지난 2004년 최초 유해요인조사 실시 후 매 3년 주기로 실시되고 있다.

Corresponding Author: Jun-Youb Lee. Industrial-Hygiene Manager, Gunsan HSE Department, Hyundai Heavy Industries Co.Ltd. Mobile: 010-9154-7136, E-mail: ergo21@hhi.co.kr

Copyright@2012 by Ergonomics Society of Korea(pISSN:1229-1684 eISSN:2093-8462). All right reserved. 
$\mathrm{H}$ 社 역시 이 주기로 근골격계질환을 유발할 수 있는 현장 의 작업유해요인에 대해 조사를 실시하고 있으며, 이에 덧붙 여 KOSHA-CODE에서 규정한 유해요인기본조사표에 추 가하여 인간공학적 유해요인조사 TOOL로 많이 사용되는 OWAS, NLE, SI, QEC 등의 정밀 조사기법을 이용한 자체조 사 역시 활발히 사용하고 있다.

본 연구에서는 여러 가지 평가기법 중 $\mathrm{H}$ 社가 대표적인 작업자세 평가기법으로 사용중인 OWAS 기법에 작업 강 도와 빈도의 개념을 추가하여 종합적인 조치 단계(Action Category; AC)를 산출하도록 자체 개발하여 사용중인 유해 요인조사 평가도구인 $\mathrm{H}-\mathrm{OWAS}$ 를 소개하고 기존 평가도구 와의 조치 단계(AC) 결과를 비교하여 어떤 평가도구가 해 당 작업자가 느끼는 실질적인 근골격계 부담 정도를 보다 잘 평가할 수 있는지 분석하였다.

\section{Method}

\subsection{HEMP(HHI Ergonomic Management Program)}

$\mathrm{HEMP}$ 란 $\mathrm{H}$ 社 노사가 수년간의 연구개발기간을 거쳐 자 체 개발한 근골격계질환 예방관리 프로그램으로써, 쾌적하고 안전한 작업환경 조성을 위하여 고안된 종합 프로그램이다.

$\mathrm{HEMP}$ 시스템은 크게 5가지 파트로 구성되어 있는데, 사 업범위가 광활한 $\mathrm{H}$ 社의 조직특성을 감안한 실행 조직의 구 성 파트와 이러한 조직을 원활하게 운영하고 전문화시켜 나 가기 위한 교육 및 홍보 파트, 그리고 근골격계질환을 유발 할 수 있는 작업유해요인을 발굴하여 개선해 나가기 위한 인간공학적 작업장 관리 파트, 실제 근골격계질환의 증상을 호소하는 종업원에게 체계적인 의료서비스를 제공하기 위해 만든 의학적 관리 파트 및 이 모든 프로그램이 원활하고 발 전적으로 유지되어 나가기 위한 Feed-back 역할을 하는 시스템 평가 파트로 구성되어져 있다.

조직구성 및 교육 파트의 경우 매년 근골격계질환 부담작 업을 보유하고 있는 전 생산/지원부서 추진위원을 선임(조직 구성)하여 전문화 교육을 실시하고 부서 전달교육(교육/홍 보)을 통해 예방관리 활동을 실시하게 된다. 또한 증상 호소 자나 징후가 보이는 작업자의 경우 사내 건강증진센터를 이 용한 조기 치료(의학적 관리)를 적극 유도하고 이러한 일련 의 활동에 대해 전담팀이 직접 현장을 방문하여 기술지원 및 운영 평가(시스템 평가)를 실시하게 된다.

$\mathrm{HEMP}$ 시스템의 가장 핵심인 인간공학적 작업장 관리의 경우 산업안전보건법 제 24 조에 의거 실시하는 법정 유해요 인조사와 더불어 OWAS, NLE, QEC, SI 등의 정밀 평가도 구를 활용하여 매월 부서별 1건 이상의 자체 유해요인조사
를 실시하고 그 결과에 따라 작업환경개선을 실시토록 하고 있다. 이러한 자체 유해요인조사를 통해 $\mathrm{H}$ 社의 사업장은 매 3년 주기라는 법적 틀에서 벗어나 정기적이고 일상적인 유 해요인조사와 문제점 발굴 및 작업환경개선이 이루어지도록 한 것이 특징이라고 할 수 있다.

\subsection{H-OWAS}

HEMP에서 사용하는 현장 유해요인에 대한 인간공학적 Risk-Assessment tool은 크게 법정 유해요인조사 방법인 KOSHA-CODE 기본 유해요인조사기법과 자체 유해요인조 사에서 사용되는 OWAS, SI, NLE, QEC 기법으로 구분된다 고 할 수 있다.

우리가 흔히 알고 있는 대표적인 작업자세 평가기법인 OWAS나 손목을 많이 사용하는 반복작업에 사용되는 SI, 중량물 취급작업을 분석하는 NLE, 영국 $\mathrm{HSE}$ 에서 개발되어 사용되는 $\mathrm{QEC}$ 의 경우 비교적 간단한 방법으로 작업현장의 근골격계질환 유해요인을 쉽게 모니터링하고 우선순위화 할 수 있다는 점에서 현장에 많이 보급되고 있으나, 각각의 기 법이 가진 특성상 실제 작업자가 느끼는 작업부담을 정량화 하여 측정하기에 한계가 있는 것이 사실이었다.

$\mathrm{H}$ 社에서는 이러한 각각의 평가기법의 단점을 극복하고자 평가자의 주관적 평가와 더불어 현장의 작업자들이 느끼는 실제 작업부하(Work load) 와 작업빈도(work frequency) 를 평가하여 종합적인 위험수준(Action Category)을 결정 할 수 있도록 보완한 평가기법을 개발하였으며, 각각의 고 유 평가기법 이름 앞에 $\mathrm{H}$ 社의 이니셜을 붙여 $\mathrm{H}-\mathrm{OWAS}$, $\mathrm{H}-\mathrm{NLE}, \mathrm{H}-\mathrm{SI}, \mathrm{H}-\mathrm{QEC}$ 라고 명명하였다.

본 연구에서는 이들 평가기법 중 작업자들의 부적절한 작 업자세를 정의하고 평가하기 위한 대표적인 관찰적 작업자 세 평가기법이며, 평가 용이성 및 단순성 등의 이유로 현장 에서의 적용성이 뛰어난 $\mathrm{H}-\mathrm{OWAS}$ 기법에 대해서만 언급 하고자 한다.

$\mathrm{H}$ 社에서 개발하여 사용중인 $\mathrm{H}-\mathrm{OWAS}$ 의 경우 단위작업 이 이루어지는 작업현장에서 평가자가 총 10 회의 등간격 평 가(통상 1 분 간격으로 총 10 분 관찰)를 실시하고, 실제 작 업현장의 생산기사 인터뷰를 통해 작업자 본인이 하고 있는 작업의 작업 강도와 빈도를 평가하게 함으로써 평가자의 주 관적 평가를 배제시켰으며, OWAS 기법의 단점으로 인식되 었던 작업자세를 제외한 위험요인이 평가에 반영될 수 있도 록 개선시킨 평가기법이다.

본 연구에서는 지난 2004년부터 실시한 근골격계 부담작 업 유해요인조사 시 사용되었던 OWAS 평가결과와 동일한 작업에 대해 $\mathrm{H}-\mathrm{OWAS}$ 로 평가된 결과를 비교해 봄으로써 통계적으로 유의한 차이가 있는지를 살펴보고자 하였고, 주 
관적인 작업부하 평가 시 많이 사용되고 있는 RPE(Ratings of Perceived Exertion) 조사를 병행함으로써 어떤 기법의 평가결과가 실제 그 작업을 하고 있는 작업자가 느끼는 주 관적인 작업피로도를 더 잘 반영하고 있는지 살펴보고자 하 였다.

\subsubsection{Quantification of work load and frequency}

작업부하 및 빈도는 $\operatorname{KOSHA~GUIDE}(\mathrm{H}-9-2011)$ 의 유 해요인기본조사 중 '작업조건조사(인간공학적인 측면을 고 려한 조사) 2 단계 각 작업별 작업부하 및 작업빈도'의 기준 을 준용하여 총점수를 산출하고 이를 4 단계로 분류하였다 (Table 1, 2).

Table 1. Work load and frequency

\begin{tabular}{c|c|c|c}
\hline Work load & Score & Frequency & Score \\
\hline Very light & 1 & $2 \sim 3$ time/month & 1 \\
\hline Light & 2 & $2 \sim 3$ time/week & 2 \\
\hline Somewhat hard & 3 & $1 \sim 2 \mathrm{hr} /$ day & 3 \\
\hline Hard & 4 & $2 \sim 4 \mathrm{hr} /$ day & 4 \\
\hline Very hard & 5 & $4 \mathrm{hr} \sim /$ day & 5 \\
\hline
\end{tabular}

Table 2. Risk-level of work condition

\begin{tabular}{c|c|c}
\hline $\begin{array}{c}\text { Multiplied score } \\
\text { (work load } \times \text { frequency) }\end{array}$ & $\begin{array}{c}\text { Modified } \\
\text { score }\end{array}$ & Risk-level \\
\hline $1 \sim 5$ & 1 & Low \\
\hline $6 \sim 10$ & 2 & Moderate \\
\hline $11 \sim 19$ & 3 & High \\
\hline $20 \sim 15$ & 4 & Very high \\
\hline
\end{tabular}

\subsubsection{OWAS}

일반적인 OWAS 평가기법으로, 해당 작업자의 자세를 일 정간격으로 관찰하여 허리, 팔, 다리, 하중/힘에 해당하는 코 드를 기록하여 근골격계에 미치는 영향에 따라 4 가지 조치 단계(Action Category; $\mathrm{AC}$ ) 로 분류하였다(Table 3).

$\mathrm{H}$ 社 OWAS 기법은 평가자의 주간적 판단오류를 최소화

Table 3. OWAS AC

\begin{tabular}{c|c}
\hline Action categories & Action required \\
\hline 1 & No action \\
\hline 2 & Action in the near future \\
\hline 3 & Action as soon as possible \\
\hline 4 & Action immediately \\
\hline
\end{tabular}

하고 단위작업 전반에 걸친 균형적 평가를 유지하기 위해 등간격 랜덤 샘플링 방식의 10회 측정을 시행하여, 그 평균 $\mathrm{AC}$ 값을 $\mathrm{OWAS}$ 평가의 $\mathrm{AC}$ 값으로 환산하였다.

\subsubsection{Final action category}

$\mathrm{H}-\mathrm{OWAS}$ 에서는 우선 작업조건 평가로 산출한 총점수 (작업부하 X작업빈도)에 의한 작업조건 위험도 수준(1 4) 과 기존 OWAS 평가로 얻는 $\mathrm{AC}(1 \sim 4)$ 의 값을 곱하여 종합 점수를 산출하고 이를 다시 최종 Action Category 4단계(수 준)로 분류하여 종합 평가결과를 산출하였다. 종합 평가결과 에 따른 조치 내용은 기존 OWAS에서 제시하고 있는 4단계 내용을 그대로 준용하였다(Table 4).

Table 4. Total Evaluation

\begin{tabular}{c|c|c|c|c|c|c}
\hline $\begin{array}{c}\text { Modified } \\
\text { score }\end{array}$ & $\begin{array}{c}\text { OWAS } \\
\text { A. C }\end{array}$ & $\begin{array}{c}\text { Final } \\
\text { score }\end{array}$ & $\begin{array}{c}\text { Final } \\
\text { AC }\end{array}$ & $\begin{array}{c}\text { Action } \\
\text { Required }\end{array}$ \\
\hline 1 & 1 & & 1 & 1 & $\begin{array}{c}\text { No } \\
\text { action }\end{array}$ \\
\hline 2 & $\mathrm{X}$ & 2 & $=$ & $2,3,4$ & 2 & $\begin{array}{c}\text { Action in } \\
\text { the near future }\end{array}$ \\
\hline 3 & 3 & & $6,8,9$ & 3 & $\begin{array}{c}\text { Action as } \\
\text { soon as possible }\end{array}$ \\
\hline 4 & & 4 & & 12,16 & 4 & $\begin{array}{c}\text { Action } \\
\text { immediately }\end{array}$ \\
\hline
\end{tabular}

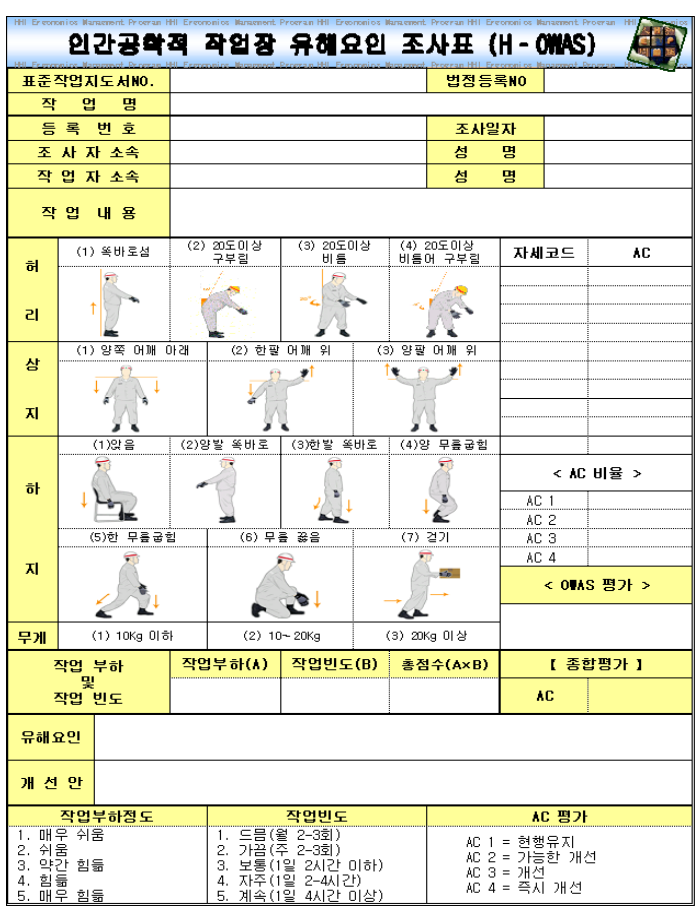

Figure 1. H-OWAS 


\subsection{Borg's scale(RPE: Ratings of Perceived Exertion)}

본 논문에서는 OWAS와 $\mathrm{H}-\mathrm{OWAS}$ 의 평가결과에 대한 비교분석을 위하여 같은 단위작업에 종사하는 작업자를 대 상으로 Borg's Scale을 이용한 RPE(Ratings of Perceived Exertion) 수준 평가를 실시하였다. 통상 Borg's Scale에서 사용하는 척도는 1 10을 기준으로 삼는 방식과 6 20을 기 준으로 삼는 두 가지 방식이 많이 사용되고 있는데 본 연구 에서는 실제 작업자의 작업피로도를 간접 측정하는 것이 목적이었기에 6 20(안정 시 Heart-rate 60-Maximum Heart-rate 200에서 착안) scale을 기준으로 하는 방법을 채택하여 실험을 실시하였다.

본 연구에서 각각의 데이터에 대한 결과 분석을 위하여 사 용한 통계프로그램은 MINITAB 15.0 Version(한글판)이다.

\section{Results}

\subsection{Comparison of the result between OWAS and H-OWAS}

같은 작업에 대해 두 가지 평가기법(OWAS와 $\mathrm{H}-$ OWAS)으로 평가한 결과를 비교분석하기 위하여 H社 내업 (완성된 배의 단위 블록을 생산하는) 부문 40개 근골격계 부담작업(동일한 표준작업지도서로 작업되는 단위작업을 의 미함)에 대해 OWAS와 H-OWAS를 이용하여 인간공학적 정밀 평가를 실시하고 $\mathrm{AC}$ 값의 차이를 통계적으로 분석(쌍 체 $\mathrm{t}$ 검정 $)$ 하였다.

또한, 각 단위 작업별로 3 명씩 선별된 대표 작업자 120 (40개 작업*3人)명에 대해 Borg's Scale을 이용한 RPE (Ratings of Perceived Exertion) 수준 평가를 통하여 작업 자들이 그 작업을 수행함에 있어 실제 느끼는 작업부하에 대 한 자각 정도를 평가한 후(각 작업별 $\mathrm{RPE}$ 대표값은 작업자 3 명에 대한 평균값을 사용함) OWAS와 $\mathrm{H}-\mathrm{OWAS}$ 평가결 과에 대한 회귀분석을 실시하여 어떤 평가도구의 결과가 작 업자들이 느끼는 실제 작업부하의 자각 정도를 더욱 잘 설 명하는지 분석하고자 하였다(모형 1: 반응변수: RPE, 예측 변수: OWAS, 모형 2: 반응변수: RPE, 예측변수: $\mathrm{H}-\mathrm{OWAS}$ ).

40개 단위작업에 대한 OWAS, H-OWAS 평가결과 및 $\mathrm{RPE}$ 설문조사 결과는 아래의 <Table 5>에서 보는 바와 같 다(지문 관계상 모든 데이터 값 나열은 생략함).

간략히 정리하면 OWAS 기법의 경우 40 개 작업에 대해 $\mathrm{AC}$ 값이 2에서 3 사이에 주로 분포하였고, $\mathrm{H}-\mathrm{OWAS}$ 의 경 우 2 에서 4 사이에 분포하여 차이점이 있었으며, $\mathrm{RPE}$ 평균 값의 경우 최소 9.67 부터 최대 15.67 까지 다양하게 결과가
나타났다.

Table 5. Result of ergonomic assessment (OWAS, H-OWAS, RPE)

\begin{tabular}{c|c|c|c}
\hline \multirow{2}{*}{ 작업 } & \multicolumn{2}{|c|}{ AC } & \multirow{2}{*}{$\begin{array}{c}\text { RPE } \\
\text { (Average) }\end{array}$} \\
\cline { 2 - 3 } & OWAS & H-OWAS & 9.67 \\
\hline Task 1 & 2 & 2 & 11.33 \\
\hline Task 2 & 2 & 3 & 15.33 \\
\hline Task 3 & 3 & 3 & 16 \\
\hline Task 4 & 3 & 4 & 14.33 \\
\hline Task 5 & 3 & 2 & $\cdots$ \\
\hline$\cdots$ & $\cdots$ & $\cdots$ & 14.67 \\
\hline Task 40 & 2 & 3 & \\
\hline
\end{tabular}

\subsection{Results of statistical analysis}

앞에서 살펴본 바와 같이 40개 단위작업에 대한 각각의 평가기법 및 $\mathrm{RPE}$ 설문조사 결과를 통계 프로그램을 이용 하여 분석한 결과는 아래의 Table 6 과 Table 7 에 나타나 있다.

Table 6. Result of the paired t-test

\begin{tabular}{c|c|c|c|c}
\hline & $\mathrm{N}$ & Mean & StDev & $p$-value \\
\hline OWAS & 40 & 2.475 & 0.5057 & \multirow{2}{*}{0.027} \\
\hline H-OWAS & 40 & 2.700 & 0.5639 & \\
\hline Difference & 40 & -0.225 & 0.6197 & \\
\hline
\end{tabular}

Table 7. Result of Regression Analysis on OWAS and H-OWAS for RPE

\begin{tabular}{c|c|c|r|c|c|c}
\hline \multirow{2}{*}{ Model } & \multicolumn{5}{|c|}{ ANOVA } & \multirow{2}{*}{$\mathrm{R}^{2}$} \\
\cline { 2 - 7 } & Source & DF & \multicolumn{1}{c|}{ MS } & $F$ & $P$ & \\
\hline RPE- & Center & 1 & 21.934 & 4.86 & 0.034 & \multirow{2}{*}{$11.3 \%$} \\
\cline { 2 - 6 } OWAS & Error & 38 & 4.512 & & & \\
\hline \multirow{2}{*}{$\begin{array}{c}\text { RPE- } \\
\text { H-OWAS }\end{array}$} & Center & 1 & 39.626 & 9.79 & 0.003 & \multirow{2}{*}{$20.5 \%$} \\
\cline { 2 - 6 } & Error & 38 & 4.046 & & & \\
\hline
\end{tabular}

주요 데이터에 대한 분석결과 OWAS와 $\mathrm{H}-\mathrm{OWAS}$ 기법 으로 평가한 각각의 단위작업에 대한 위험도에 유의한 차이 가 있는 것으로 나타났으며 $(p$ value $=0.027), \mathrm{H}$-OWAS 의 평가결과가 OWAS로 평가한 결과에 비해 대체로 위험성 이 높은 것으로 나타났다(Table 6).

또한, 본 연구에서 어떤 평가기법이 실제 작업현장의 작업 위험도 및 작업피로도를 더 적절하게 표현하고 있는지를 알 
아보기 위해 실시한 RPE 값과 OWAS 및 $\mathrm{H}-\mathrm{OWAS}$ 평가 결과인 Action Category 값을 회귀분석을 통하여 비교분석 한 결과, 두 평가도구 모두 $\mathrm{RPE}$ 값과 유의성을 가진다고 할 수 있었으며 (모형 1 회귀식 $p$-value $=0.034$, 모형 2 회 귀식 $p$-value $=0.003)$, 각 모형별 결정계수 $\left(\mathrm{R}^{2}\right)$ 를 비교 한 결과 $\mathrm{H}-\mathrm{OWAS}$ 를 이용한 평가결과가 OWAS를 이용한 평가결과 보다 작업자의 자각에 의한 작업부하(또는 작업피 로도)를 훨씬 더 잘 설명하는 것으로 관찰되었다(Table 7).

이는 40개의 단위작업이 가지는 각각의 작업특성 상, 작 업자세 및 일부 중량물 취급형태를 제외한 부분에서 평가요 소가 누락될 수 있는 OWAS 기법의 단점을 작업부하 및 작 업빈도 점수에 따른 작업조건 위험도를 평가요소에 반영시 킨 $\mathrm{H}-\mathrm{OWAS}$ 기법의 특징이 실제 근골격계질환을 유발할 수 있는 현장의 작업유해요인 및 Risk Factor들을 평가에 더 잘 반영한다는 결과로 해석할 수 있다.

\section{Conclusion}

본 논문에서는 지난 2002년 $\mathrm{H}$ 社 노사가 근골격계질환 예 방을 위하여 공동으로 개발한 $\mathrm{HEMP}$ 시스템에 대한 전반 적인 이해와 더불어 $\mathrm{HEMP}$ 의 주요 구성요소인 인간공학적 인 작업장 관리를 함에 있어 현장의 주요 위험요인에 대한 Risk-Assessment 방법론에 대해 소개하고자 하였다.

또한 인간공학적 Risk-Assessment 툴(Tool)로 많이 사 용되고 있는 OWAS, NLE, SI, QEC 등의 평가기법에 추가 하여 작업빈도와 작업부하에 대한 요소를 위험성 평가요소 에 반영하여 $\mathrm{H}$ 社가 독자 개발한 평가기법 중 조선업에서 가 장 많이 사용되고 있는 $\mathrm{H}-\mathrm{OWAS}$ 기법을 소개하였다.

$\mathrm{H}-\mathrm{OWAS}$ 의 경우 본 연구의 결과에서 살펴 볼 수 있듯이 기존 OWAS 기법이 가진 단점 (작업자세를 제외한 부분의 평가요소가 미흡하고, 평가자의 주관적인 판단이 과다하게 반영되는 점)을 극복하고자, 총 10회의 랜덤 샘플링 방법으 로 작업상황을 1 차 평가한 후 작업부하 및 작업빈도를 크로 스로 평가하는 2 차 평가과정을 거쳐 종합적인 평가결과를 도출하기 때문에 실제 작업현장에 종사하는 작업자들이 느 끼는 작업피로도(또는 작업위험성)를 더 잘 설명하는 것으 로 나타났다.

본 평가도구는 $\mathrm{H}$ 社 사업장 인간공학 전담팀의 다년간에 걸친 자체 평가 및 분석에 따른 판단에 근거하여 고안된 도 구로, 작업장 근골격계질환 부담작업에 대하여 보다 현실적 이고 실용적인 평가를 가능하게 했다는데 의의가 있다고 할 수 있다. 단, 본 연구에서 도출된 통계적 유의성은 단일 작업 장 내의 일부 데이터에 기초하여 산출된 결과로써 이를 일반 화 시키기 위해서는 보다 다양한 작업현장(예를 들어, 조선
업을 제외한 제조업 등)에서의 많은 데이터 수집 및 추가 연 구가 필요할 것으로 판단되며, 이와 더불어 작업현장의 현실 적인 제약으로 인해 작업자의 작업피로도를 측정하는데 있 어 간접측정방식 $(\mathrm{RPE})$ 을 사용했던 경험을 바탕으로 직접적 인 작업피로도 측정방식 (ex: EMG, Heart-rate 측정, 기타 작업생리학적 접근 등)을 이용한 후행 연구가 필요할 것으 로 생각된다.

\section{References}

Borg, G. "Psychophysical bases of perceived exertion", Medicine and Science in Sports and Exercise, 14(5), pp.377-381, 1982.

Kim, C. H. and Fernandez, J. E., "Psychophysical frequency for a drilling task", International Journal of Industrial Ergonomics, 12, pp. 209 $-218,1993$.

Lee, J. Y., "A Study of workload change at varying working posture, work/rest ratio and task type in an automobile assembly task", Journal of the Society of Korea Industrial and Systems Engineering, Vol. 24, No. 69, 2001.

Lee, J. Y. and Seo, H, S., "The Method of the risk assessment on ergonomics in large-scale corporations", Spring Conference of Ergonomics Society of Korea, 2007.

\section{Author listings}

Jun-Youb Lee: ergo21@hhi.co.kr

Highest degree: M.S, Department of Industrial Engineering, University of Incheon

Position title: Industrial-Hygiene Manager, Gunsan HSE Department, Hyundai Heavy Industries Co.Ltd

Areas of interest: Work-Physiology, Risk Management, Man-Machine system

Hyun-Soo Seo: hsseo@hhi.co.kr

Highest degree: M.S, Department of Industrial Engineering, Hanyang University

Position title: Industrial-Hygiene Manager, HSE Department, Hyundai Heavy Industries Co.Ltd

Areas of interest: Work-Physiology, Risk Management, HCI

Date Received : 2011-12-22

Date Revised :2012-01-16

Date Accepted : 2012-01-16 